Journal of Animal and Veterinary Advances 10 (9): 1227-1231, 2011

ISSN: $1680-5593$

(C) Medwell Journals, 2011

\title{
Construction of Lentivirus-Mediated Retinal Pigment Epithelium Cells Stably Expressing Basic Fibroblast Growth Factors
}

\author{
Shi-Wei Huang, Guan-Fang Su and Hong Wu \\ Department of Ophthalmology, The Second Hospital of Jilin University, \\ Changchun 130041, Jilin Province, China
}

\begin{abstract}
The transplantation of Retinal Pigment Epithelium (RPE) cells was a potential therapeutic strategy in some retinal diseases. To construct RPE cells capable of stably and efficiently expressing basic Fibroblast Growth Factors (bFGF) using a lentivirus system, we performed the current study. The genes of bFGF were cloned into a lentivirus expression vector then the recombinant lentivirus was packaged and harvested. RPE cells were infected and the expression of the bFGF was detected by western blot. The results showed that the lentivirus vector can efficiently transfer genes into RPE cells with long-term expression. Therefore, it is likely to culture a great number of human RPE cells in vitro to provide adequate and safe low-cost donor cells for RPE cells transplantation.
\end{abstract}

Key words: Retinal pigment epithelium cell, lentivirus, basic fibroblast growth factor

\section{INTRODUCTION}

The Retinal Pigment Epithelium (RPE) is a very important component in maintaining the integrity of the retinal structure and function and the dysfunction of the RPE cells is closely related to the nosogenesis of many retinal diseases such as retinitis pigmentosa, age-related macular degeneration, etc. Many studies have shown that the transplantation of the RPE cells had a rescue effect on photoreceptor cells (Little et al., 1996; Woch et al., 2001; Algvere et al., 1994; Peyman et al., 1991). Recently, it is one of the focal point that the gene-transfected RPE cells were culture in vitro and transplanted on therapeutic strategy in some retinal diseases.

The basic Fibroblast Growth Factors (bFGF) were initially purified from bovine pituitary extracts. This growth factor is produced by many cell types and tissues and plays an important role in cell differentiation, cell proliferation, mitogenesis and angiogenesis (Bikfalvi et al., 1997). The bFGF has five isoforms of 18 , $22,22.5,24$ and $34 \mathrm{kDa}$. Although, devoid of signal peptide, $18 \mathrm{kDa} \mathrm{Bfgf}$ as the main isoform could be secreted. It acts in a paracrine and autocrine manner (Arnaud et al., 1999). The bFGF plays an important role in the development and growth of retina. At present, recognized as photoreceptor cells survival factors, bFGF is capable of promoting the survival of degenerating photoreceptor cells and rescuing the phagocytosis of RPE cells.
In this study, human RPE cells which stably express $18 \mathrm{kDa}$ bFGF have been established by a lentiviral vector system and the human RPE cells lay the foundation for providing preferable RPE cell donors for RPE cell transplantation and treating related retinal diseases.

\section{MATERIALS AND METHODS}

Main experiment materials: Lentiviral vector system (containing lentivirus expression vector pLVX-IRESZsGreen and Lenti-X HT Packaging system, Clontech), 293 T cells (Clontech), restriction enzymes and T4 DNA Ligases (TaKaRa), FuGENE ${ }^{\circledR}$ HD Transfection Reagent (Roche), human RPE cell line (from ATCC, a kind gift from Shanghai Institute of Ophthalmology), plasmids pLN-F (containing bFGF cDNA constructed and reserved by this laboratory), RIPA lysate and BCA Protein Assay (Watson Biotech inc.), mouse anti-human bFGF antibodies, GAPDH antibodies (Santa Cruz) and Super ECL Plus detection reagent (Applygen Technologies Inc.).

Construction and identification of recombinant lentivirus vectors: $A$ BamHI/EcoRI fragment of the human $b F G F$ cDNA was obtained from plasmid pLN-F. Briefly, a $490 \mathrm{bp}$ fragment containing the coding region of $\mathrm{bFGF}$ was cut out from the plasmid. After purification by gel electrophoresis, this fragment was inserted into the plasmid pLVX-IRES-ZsGreen. The constructed plasmid PLV-bFGF-IRES-ZsGreen was identified by gel electrophoresis after restriction enzyme digestion (Kpn I; Xba I and Nhe I).

Corresponding Author: Hong Wu, Department of Ophthalmology, The Second Hospital of Jilin University, Changchun 130041, Jilin Province, China 
Packaging and harvesting of lentiviruses: The 1 day before the transfection, the $293 \mathrm{~T}$ cells were trypsinized and plated the cells in six-well plate to achieve $70-80 \%$ confluency on the day of the transfection. Briefly, PLV-bFGF-IRES-ZsGreen/pLVX-IRES-ZsGreen was transfected together with Lenti-X HT packaging mix into 293 T cells using FuGENE ${ }^{\circledR}$ HD Transfection Reagent. After $8 \mathrm{~h}$ later, the culture medium was replaced. Harvesting was undertaken at $48 \mathrm{~h}$. These viruses were kept at $-80^{\circ} \mathrm{C}$ until just before use.

Infection of RPE cells with recombinant lentiviruses: The RPE cells were placed in a six-well and incubated at $37^{\circ} \mathrm{C}$, $5 \% \mathrm{CO}_{2}$ overnight. The culture medium was changed when the RPE cells were approximately $70 \%$ confluent. Then $200 \mu \mathrm{L}$ virus was added into the six-well plate. About $24 \mathrm{~h}$ after infection, another fresh culture medium was used for replacement. About $48 \mathrm{~h}$ after infection, the RPE cells were observed by a fluorescence microscope. The cells expressing ZsGreen fluorescent protein were expanded in culture.

Protein extraction and western blotting assay: The infected RPE cells (cultured for 1, 4 and 12 weeks) were lysed in RIPA lysis buffer and cocktail proteinase inhibitor. Cells lysates were centrifuged at $10000 \times \mathrm{g}$ for $3 \mathrm{~min}$ at $4^{\circ} \mathrm{C}$ and then supernatant was collected. The protein concentrations were quantitated by BCA Protein Assay kit. The uninfected RPE cells were prepared by the same method above. After SDS-PAGE, the protein were transferred to a PVDF membrane and the membrane were blocked and probed with mouse anti human monoclonal bFGF antibody in 5\% nonfat dried milk with a solution of TBS containing $0.05 \%$ Tween-20 overnight. After $30 \mathrm{~min}$ washes in TBS-0.05\% Tween-20, blots were incubated with horseradish peroxidase-conjugated secondary antibodies for $60 \mathrm{~min}$ followed by $30 \mathrm{~min}$ washes in TBS- 0.05 Tween- 20 . The protein bands were visualized by enhanced chemiluminescence detection reagents after exposure of the membrane to film for $30-60 \mathrm{sec}$.

Statistical analysis: Results were presented as mean \pm SD. Statistical significance was analyzed by t-test. The value of $\mathrm{p}<0.05$ is considered significant.

\section{RESULTS AND DISCUSSION}

The results demonstrated that the $b F G F$ gene was inserted into the plasmid without any mutation by sequencing. The expression of ZsGreen fluorescent protein could be observed clearly $24 \mathrm{~h}$ after being

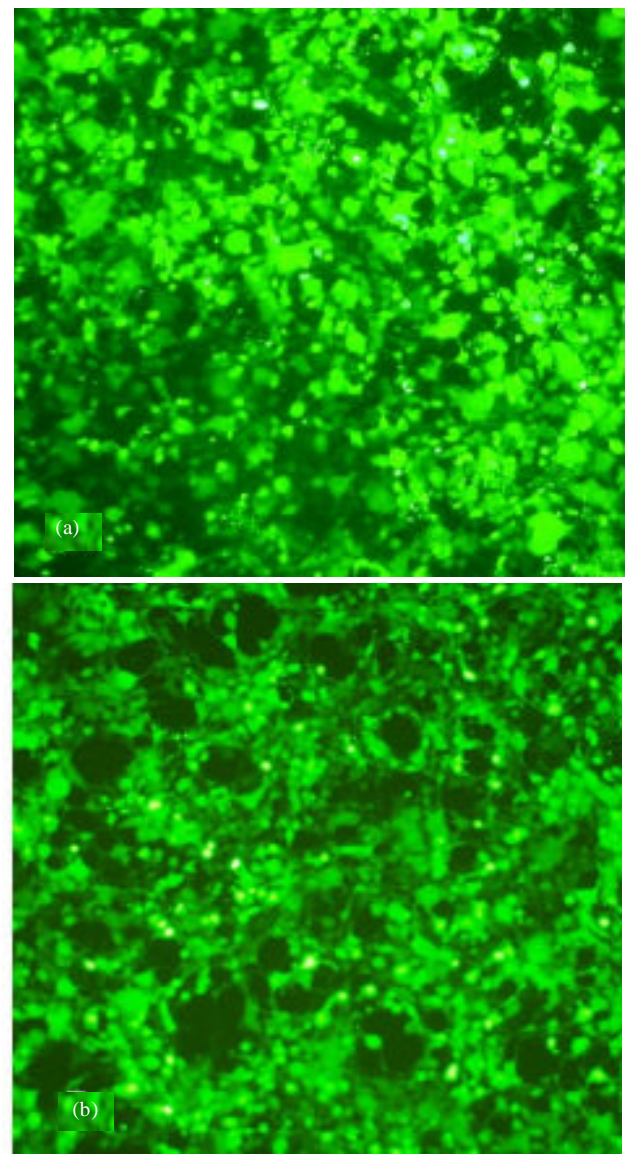

Fig. 1: $293 \mathrm{~T}$ cells were transfected by PLV-bFGF-IRESZsGreen (a) and PLVX-IRES-ZsGreen (b)

transfected (Fig. 1). We transfect $>99 \%$ of $293 \mathrm{~T}$ cells easily by the lentiviral vector systems. As shown in Fig. 2, recombinant lentiviruses infected the RPE cells efficiently and completely.

After 1, 4 and 12 weeks when RPE cells were infected by the lentiviruses carrying $b F G F$ genes, bFGF expression could be detected by Western Blot but in the control (uninfected RPE cells and the RPE cells infected by PLVX-IRES-ZsGreen), bFGF expression could not be detected (Fig. 3a). The protein levels were represented by the ratio of net density of bFGF and GAPDH (Fig. 3b). The expression level of the bFGF of the infected cells was significant compared with the control groups $(\mathrm{p}<0.01)$.

It has been reported that the retinal degeneration can be widely rescued by the injection of bFGF protein (Faktorovich et a1., 1990; LaVail et al., 1998). The intravitreally and subretinally injected bFGF protein remarkably delayed the retinal degeneration in such cases. However, there are some problems that limit the clinical application of the bFGF (e.g., the short half-life 

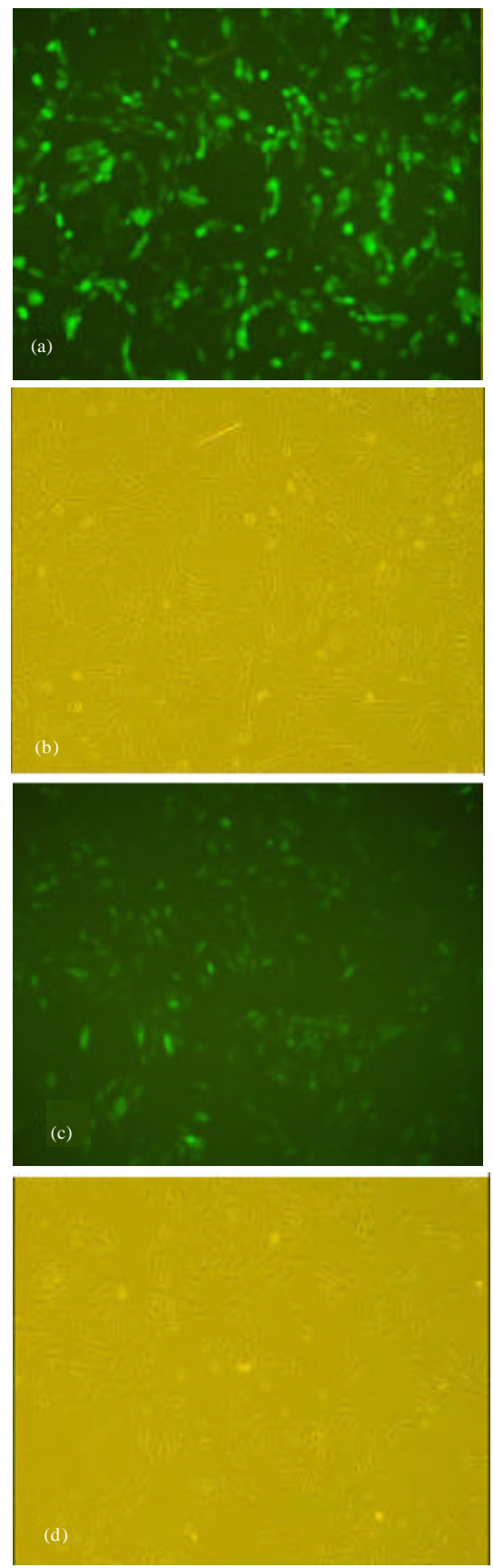

Fig. 2: RPE cells were infected by PLV-bFGF-IRESZsGreen (a, b) and PLVX-IRES-ZsGreen (c, d). Photographs were taken at $48 \mathrm{~h}$ (left: under fluorescence microscopy, right: under phase contrast microscopy)

period of the factors and the effective concentration is retained difficultly, so it is necessary to administrate many times or increase dose; related complicating diseases such as cataract, retinal vascularization and the like can be caused (Perry et al., 1995); exogenous recombinant
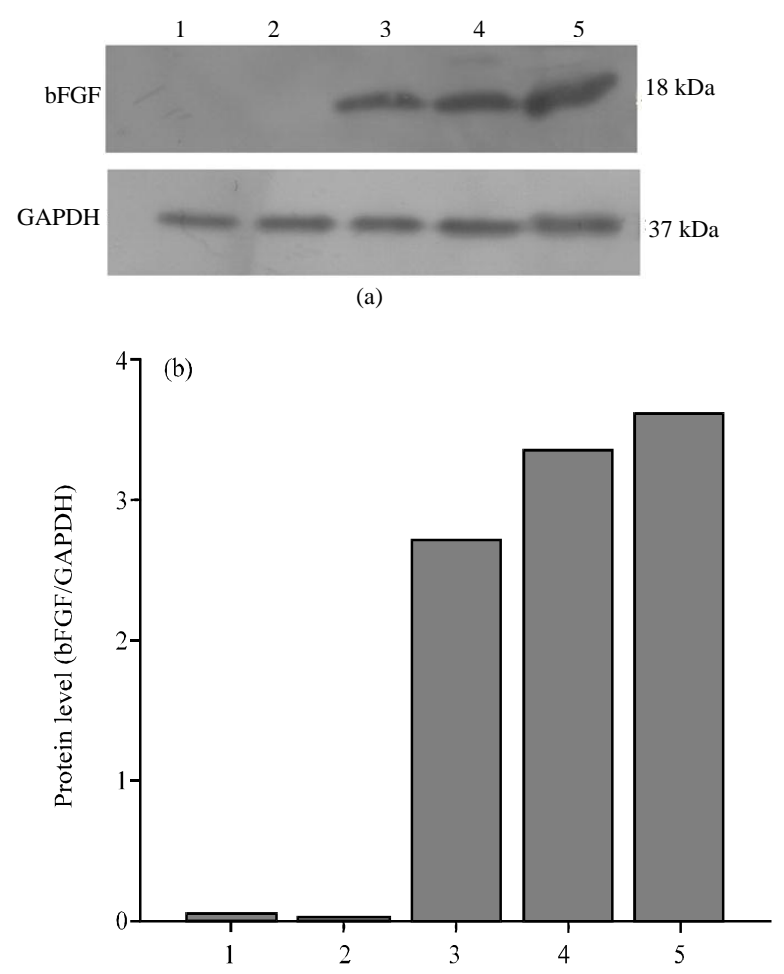

Fig. 3: The level of the expression of bFGP in RPE cells; a) Western blot result; $b$ ) The result of bGFP/GAPDH 1: RPE cells; 2 : RPE cells infected by PLVX-IRESZsGreen 3, 4 and 5: 1, 4 and 12 weeks after infection

protein is expensive). Moreover, compared with exogenous recombinant protein, endogenous protein has higher biological activity (Kang et al., 2000), researchers attempt transmitting $b F G F$ genes into eyeballs to rescue the degeneration of photoreceptors by using the gene therapy, including the electroporation method (Ogata et al., 1999), the K8/JTS-1-mediated transfection technique (Neuner-Jehle et al., 2000), recombinant adenoviral vector (Akimoto et al., 1999) and recombinant adeno-associated virus vector (Lau et al., 2000), etc. The results showed that the amount of secreted protein was much smaller than that of recombinant bFGF injected as a control however, the effect of rescue of photoreceptor cell achieved by recombinant adenovirus injection was greater than that of recombinant bFGF (Akimoto et al., 1999) and the side effects such as cataract or tumor-like cell proliferation and the like were not detected (Neuner-Jehle et al., 2000). Recent research indicated that neural retina limits the nonviral gene transfer to retinal pigment epithelium (Pitkanen et al., 2004).

Meanwhile with the development of vectors, Lentiviral vectors have become some of the most widely used vectors for fundamental biological research, functional genomics and gene therapy. The lentiviral 
vectors can stably integrate into the genome of target cells and result in persistent expression of the gene of interest. Lentiviral vectors are able to transduce dividing and non-dividing cells (Lai and Brady, 2002). Moreover, Lentiviral vectors can accommodate larger transgenes (up to 10 kilobases) compared to other vectors. Importantly, lentiviral transduction results in low intrinsic immunogenicity (Blomer et al., 1997).

Lenti-X HT Packaging system, used in this study is the fourth-generation packaging systems and it easily produces high titer lentiviral supernatants suitable for safe use. The supernatant can be used to infect target cells directly without prior concentration. We have observed stable transgene expression of a ZsGreen fluorescent protein in greater than $99 \%$ of cells $>3$ months after transduction.

The transplantation of RPE cells brought the hope of the therapy of retinal diseases caused by abnormal RPE function (Little et al., 1996; Woch et al., 2001; Algvere et al., 1994, 1997; Peyman et al., 1991). The RPE cells can release factors, e.g. bFGF and these factors can provide nutrition for the retina and promote the differentiation and survival of photoreceptors (Sheedlo et al., 1992; Aymerich et al., 2001) (according to the results of the study, the RPE cells secrete few bFGF which can not be inspected by western blot). Thus in addition to the transplantation of normal RPE cells, the transplantation of gene-transfected RPE cells not only prevent the degeneration of photoreceptors but also regulate the bFGF levels at retina which would be of wide application for treating retinal diseases (Saigo et al., 2004).

\section{CONCLUSION}

This study demonstrated that lentiviral vectors can efficiently transfer $b F G F$ genes into RPE cells with stable long-term expression in vitro. Therefore, it is likely to culture a great number of human RPE cells in vitro to provide adequate and safe low-cost donor cells for RPE cell transplantation.

\section{REFERENCES}

Akimoto, M., S. Miyatake, J. Kogishi, M. Hangai and K. Okazaki et al., 1999. Adenovirally expressed basic fibroblast growth factor rescues photoreceptor cells in RCS rats. Invest. Ophthalmol. Vis. Sci., 40: 273-279.

Algvere, P.V., L. Berglin, P. Gouras and Y. Sheng, 1994. Transplantation of fetal retinal pigment epithelium in age-related macular degeneration with subfoveal neovascularization. Graefes Arch. Clin. Exp. Ophthalmol., 232: 707-716.
Algvere, P.V., L. Berglin, P. Gouras, Y. Sheng and E.D. Kopp, 1997. Transplantation of RPE in agerelated macular degeneration: Observations in disciform lesions and dry RPE atrophy. Graefes Arch. Clin. Exp. Ophthalmol., 235: 149-158.

Arnaud, E., C. Touriol, C. Boutonnet, M.C. Gensac, S. Vagner, H. Prats and A.C. Prats, 1999. A new 34kilodalton isoform of human fibroblast growth factor 2 is cap dependently synthesized by using a nonAUG start codon and behaves as a survival factor. Mol. Cell Biol., 19: 505-514.

Aymerich, M.S., E.M. Alberdi, A. Martinez and S.P. Becerra, 2001. Evidence for pigment epitheliumderived factor receptors in the neural retina. Invest. Ophthalmol. Vis. Sci., 42: 3287-3293.

Bikfalvi, A., S. Klein, G. Pintucci and D.B. Rifkin, 1997. Biological roles of fibroblast growth factor-2. Endocr. Rev., 18: 26-45.

Blomer, U., L. Naldini, T. Kafri, D. Trono, I.M. Verma and F.H. Gage, 1997. Highly efficient and sustained gene transfer in adult neurons with a lentivirus vector. J. Virol., 71: 6641-6649.

Faktorovich, E.G., R.H. Steinberg, D. Yasumura, M.T. Matthes and M.M. LaVail, 1990. Photoreceptor degeneration in inherited retinal dystrophy delayed by basic fibroblast growth factor. Nature, 347: 83-86.

Kang, R., S.C. Ghivizzani, T.S. Muzzonigro, J.H. Herndon, P.D. Robbins and C.H. Evans, 2000. Orthopaedic applications of gene therapy: From concept to clinic. Clin. Orthopaedics Related Res., 374: 324-337.

LaVail, M.M., D. Yasumura, M.T. Matthes, C. LauVillacorta, K. Unoki, C.H. Sung and R.H. Steinberg, 1998. Protection of mouse photoreceptors by survival factors in retinal degenerations. Invest. Ophthalmol. Vis. Sci., 39: 592-602.

Lai, Z. and R.O. Brady, 2002. Gene transfer into the central nervous system in vivo using a recombinanat lentivirus vector. J. Neurosci. Res., 67: 363-371.

Lau, D., L.H. McGee, S. Zhou, K.G. Rendahl, W.C. Manning, J.A. Escobedo and J.G. Flannery, 2000. Retinal degeneration is slowed in transgenic rats by AAV-mediated delivery of FGF-2. Invest. Ophthalmol. Vis. Sci., 41: 3622-3633.

Little, C.W., B. Castillo, D.A. DiLoreto, C. Cox, J. Wyatt, C. del Cerro and M. del Cerro, 1996. Transplantation of human fetal retinal pigment epithelium rescues photoreceptor cells from degeneration in the Royal College of Surgeons rat retina. Invest. Ophthalmol. Vis. Sci., 37: 204-211.

Neuner-Jehle, M., L.V. Berghe, S. Bonnel, Y. Uteza and F. Benmeziane et al., 2000. Ocular cell transfection with the human basic fibroblast growth factor gene delays photoreceptor cell degeneration in RCS rats. Hum. Gene. Ther., 11: 1875-1890. 
Ogata, N., M. Nishizawa, A. Ando and M. Uyama, 1999. Transfection of basic fibroblast growth factor (bFGF) gene or bFGF antisense fene into human fetinal pigment epithelial cells. Graefes Arch. Clin. Exp. Ophthalmol., 237: 678-684.

Perry, J., J. Du, H. Kjeldbye and P. Gouras, 1995. The effects of bFGF on RCS rat eyes. Curr. Eye Res., 14: 585-592.

Peyman, G.A., K.J. Blinder, C.L. Paris, W. Alturki, N.C. Jr. Nelson and U. Desai, 1991. A technique for retinal pigment epithelium transplantation for age-related macular degeneration secondary to extensive subfoveal scarring. Ophthalmic. Surg., 22: $102-108$.
Pitkanen, L., J. Pelkonen, M. Ruponen, S. Ronkko and A. Urtti, 2004. Neural retina limits the nonviral gene transfer to retinal pigment epithelium in an in vitro bovine eye model. Am. Assoc. Pharm. Sci. J., 6: 72-80.

Saigo, Y., T. Abe, M. Hojo, H. Tomita, E. Sugano and M. Tamai, 2004. Transplantation of transduced retinal pigment epithelium in rats. Invest. Ophthalmol. Vis. Sci., 45: 1996-2004.

Sheedlo, H.J., L. Li and J.E. Turner, 1992. Effects of RPEcell factors secreted from permselective fibers on retinal cells in vitro. Brain Res., 587: 327-337.

Woch, G., R.B. Aramant, M.J. Seiler, B.T. Sagdullaev and M.A. McCall, 2001. Retinal transplants restore visually evoked responses in rats with photoreceptor degeneration. Invest. Ophthalmol. Vis. Sci., 42: 1669-1672. 